\title{
Durable and Washable Antibacterial Copper Nanoparticles Bridged by Surface Grafting Polymer Brushes on Cotton and Polymeric Materials
}

\author{
Chufeng Sun, ${ }^{1} \mathrm{Yi} \mathrm{Li}^{2}{ }^{2} \mathrm{Zhi} \mathrm{Li}^{3}{ }^{3}$ Qiong Su, ${ }^{1}$ Yanbin Wang, ${ }^{1}$ and Xuqing Liu ${ }^{2}{ }^{2}$ \\ ${ }^{1}$ School of Chemical Engineering, Northwest Minzu University, Lanzhou 730030, China \\ ${ }^{2}$ School of Materials, University of Manchester, Oxford Road, Manchester M13 9PL, UK \\ ${ }^{3}$ College of Textiles and Garments, Southwest University, Chongqing 400715, China \\ Correspondence should be addressed to Xuqing Liu; xuqing.liu@manchester.ac.uk
}

Received 14 September 2017; Accepted 27 November 2017; Published 29 January 2018

Academic Editor: Rajesh R. Naik

Copyright (c) 2018 Chufeng Sun et al. This is an open access article distributed under the Creative Commons Attribution License, which permits unrestricted use, distribution, and reproduction in any medium, provided the original work is properly cited.

\begin{abstract}
To increase the durability of antibacterial coating on cotton and polymeric substrates, surface initiated grafting polymer brushes are introduced onto the substrates surface to bridge copper nanoparticles coatings and substrate. The morphologies of the composites consisting of the copper nanoparticles and polymer brushes were characterized with scanning electron microscopy (SEM). It was found that copper nanoparticles were uniformly and firmly distributed on the surfaces of the substrates by the polymer brushes; meanwhile, the reinforced concrete-like structures were formed in the composite materials. The substrates coated by the copper nanoparticles showed the efficient antibacterial activity against Staphylococcus aureus (S. aureus) and Escherichia coli (E. coli) even after washing by 30 cycles. The copper nanoparticles were tethered on the substrates by the strong chemical bonds, which led to the excellent washable fitness and durability. The change of the phase structure of the copper was analyzed to investigate the release mechanism of copper ions.
\end{abstract}

\section{Introduction}

Bacterial infection, as a major issue, has been arising from bacterial adhesion, growth, and proliferation on surfaces [13]. A considerable number of research works have been conducted with the aim of preparing the novel antibacterial materials [4]. The antibacterial properties of gold and silver are well known, and the effectiveness in reducing the growth of several microorganisms has been reported [5-7]. Because of high cost of silver and gold, material chemists have focused their attention on exploring the possibility of using copper as the ultimate antimicrobial agent [8-11].

However, the current techniques, such as spinning and surface coating, for fabricating medical antimicrobial textile using nanoparticles have different limitations. Spinning method works well with the synthetic fiber, but the nature fiber such as cotton is impossible to be melted and spun. Surface coating via physical absorption is the other common technique to obtain the antimicrobial performance.
However, the adhesion between the substrate and coating by physical absorption is weak. In composites discipline, Young's modulus of copper is $110 \sim 128 \mathrm{GPa}$, and yet that of the polymeric materials, such as nylon, polypropylene, and polyethylene terephthalate, is around $1 \sim 4 \mathrm{GPa}$. This intrinsic distinction causes that the copper nanoparticles could hardly be coated on the polymer surface to form composites with structural integrity [12]. The most common process of conferring the copper nanoparticles to fabrics materials is utilizing some adhesion and cross-linked agents, such as acrylate and polyurethane, to form a composite coating on the substrate $[13,14]$. Consequently, only the exposed copper nanoparticles on the external surface can be released and show antibacterial property subsequently; meanwhile the majority of nanoparticles are blocked in the inner of composites by adhesion agents $[15,16]$.

Polymer brushes assisted copper electroless deposition methods have been demonstrated to be a powerful tool in preparing copper nanoparticles on polymer 


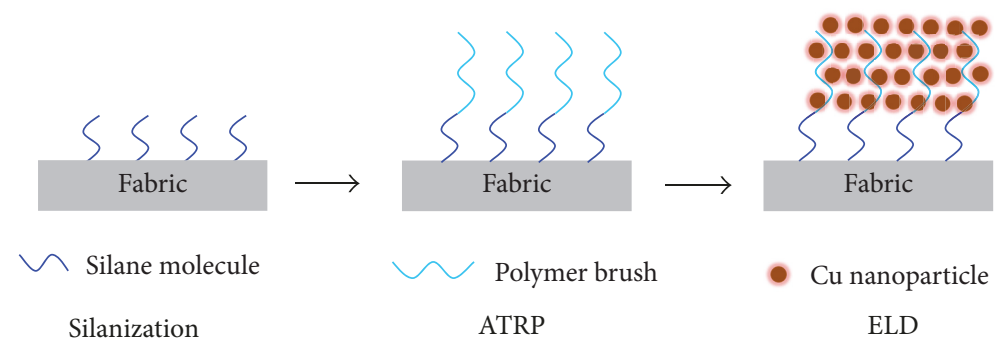

FIGURE 1: Schematic illustration of the coating-fabricated process linking fabric and free standing nano copper particles by polymer brush.

substrates [17-19]. Heuristically, the free release and reinforced concrete-like antibacterial system were designed by chemical bonds tethered copper nanoparticles on cotton and polyester substrates using atom transfer radical polymerization (ATRP) and electroless deposition (ELD). The cheaper copper nanoparticles with high effectiveness and long durable properties could match the requirement of antibacterial materials for industrial production. In this paper, cotton is chosen as the substrate material because it is used more widely than any other natural fiber, and polyester, as a typical polymeric material, is chosen as another substrate. Meanwhile, two kinds of materials are the major medical materials used in healthcare and medical fields. The antibacterial properties of copper nanoparticles covalently bonded on cotton and polymeric materials by surface grafted polymer brushes were investigated.

\section{Experimental}

2.1. Materials. All chemicals were purchased from Aldrich. The inhibitor in the monomers, [2-(methacryloyloxy) ethyl] trimethylammonium chloride (METAC), was removed by elution through a neutral alumina plug before use. Cotton yarns and fabric used in the experiments were pretreated by singeing, desizing, scouring, bleaching, and mercerizing. After that, they were cleaned with water, ethanol, and toluene to remove any possible impurities before being used, dried at room temperature, and cured at $105^{\circ} \mathrm{C}$ for $24 \mathrm{~h}$.

2.2. Fabrication. Cotton or polyester substrates were immersed in an anhydrous toluene solution of $5 \mathrm{mM} 3$-(trichlorosilyl)propyl 2-bromo-2-methylpropanoate as ATRP initiator in order for the hydroxyl groups of cellulose surface to react with the silane molecules by a condensation reaction. Then the substrates were rinsed for several times with anhydrous toluene and dichloromethane to remove the excess initiator and byproduct and at last dried at $50^{\circ} \mathrm{C}$ under vacuum for $2 \mathrm{~h}$. The initiator-modified cotton substrates were obtained.

$10 \mathrm{~mL}$ of $4.5 \mathrm{~mol} \mathrm{~L}^{-1}$ [2-(methacryloyloxy)ethyl]trimethylammonium chloride (METAC) methanol solution was prepared for the polymerization reaction under nitrogen flow at $20^{\circ}$ C. 2,2-Dipyridyl (0.48 g), Cu(I)Br (0.12 g), and $\mathrm{Cu}(\mathrm{II}) \mathrm{Br}_{2}$ $(0.019 \mathrm{~g})$ were mixed into the above solution by stirring under nitrogen flow. The initiator-modified substrates were sealed in Schlenk tubes, degassed by nitrogen, and left at $20^{\circ} \mathrm{C}$ under nitrogen. Then enough polymerization solution was syringed into each Schlenk tube for submerging each sample completely. The substrates were kept in the solution for polymerization reaction. After polymerization, the samples were removed, cleaned thoroughly by methanol and water, respectively, and then dried under nitrogen flow.

The plating bath for copper electroless deposition was composed of 1:1 mixture of freshly prepared solutions A and B. Solution A was composed of $12 \mathrm{~g} \mathrm{~L}^{-1} \mathrm{NaOH}, 13 \mathrm{~g} \mathrm{~L}^{-1}$ $\mathrm{CuSO}_{4} \cdot 5 \mathrm{H}_{2} \mathrm{O}$, and $29 \mathrm{~g} \mathrm{~L}^{-1} \mathrm{KNaC}_{4} \mathrm{H}_{4} \mathrm{O}_{6} \cdot 4 \mathrm{H}_{2} \mathrm{O}$. Solution $\mathrm{B}$ was $9.5 \mathrm{~mL} \mathrm{~L}^{-1} \mathrm{HCHO}$. Distilled water was used to prepare the plating solution. After electroless deposition, the copper nanoparticle coatings bridged by surface grafted polymer brushes were obtained on the cotton or polyester substrates, expecting the remarkable antibacterial abilities. Figure 1 shows the preparation process of the $\mathrm{Cu}$ nanocomposite coating on the fabrics.

2.3. Characterization. The structure of cotton and modified cotton was investigated by Nicolet iS10 FTIR spectrometer. The morphologies of the samples were observed using a field emission scanning electron microscope (FESEM, JEOL JSM7500). The phase structures of the samples were analyzed on X-ray diffraction (XRD) (Rigaku D/max-2400, Cu K radiation, $\lambda=0.1541 \mathrm{~nm}$.). The antimicrobial activity of copper nanoparticles loaded with cotton fabrics against four bacteria, two Gram-positive (S. aureus ATCC 25923 and B. bombysepticus) and two Gram-negative (E. coli ATCC 25922 and $S$. maicesceis), was evaluated by radial diffusion assay [20]. A single colony of each bacterial strain was inoculated into $5 \mathrm{ml}$ of $\mathrm{LB}$ at $37^{\circ} \mathrm{C}$ overnight, and about $50 \mu \mathrm{l}$ of culture was then added to $5 \mathrm{ml}$ of LB for $3-4 \mathrm{~h}$ of incubation until $\mathrm{OD}_{600}$ reached 0.4 . Approximately $1 \mathrm{ml}$ culture was mixed with $100 \mathrm{ml}$ of sterile $\mathrm{LB}$ agar at around $55^{\circ} \mathrm{C}$, and the mixture was aliquot in Petri dishes. The entire specimen was cut into $5 \mathrm{~mm}$ diameter and incubated overnight at $37^{\circ} \mathrm{C}$. The $\mathrm{Cu}$ ions release was examined using the standard atomic absorption spectroscopy (AAS) (Z-8000, Hitachi).

\section{Result and Discussion}

3.1. FTIR Analyses of Cotton and Modified Cotton. Fourier transform infrared spectroscopy (FTIR) was used to demonstrate the structure of unmodified cotton and PMETAC modified cotton in Figure 2. The new peak at $872 \mathrm{~cm}^{-1}$ and strengthened peak at $1708 \mathrm{~cm}^{-1}$ in PMETAC modified cotton are attributed to quaternary ammoniums groups $\left(\mathrm{QA}^{+}\right)$and the carbonyl groups in PMETAC, confirming the success 


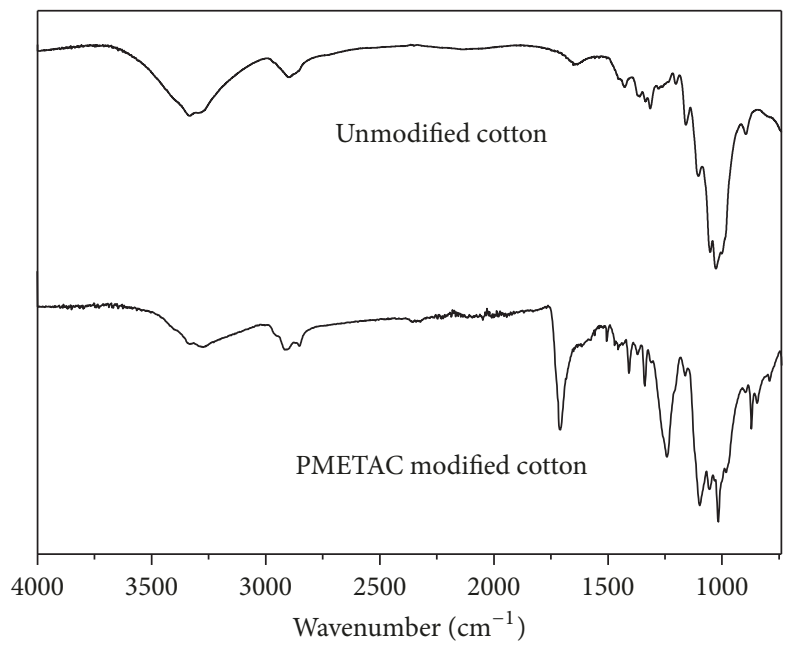

FIGURE 2: FTIR spectrum of unmodified cotton and PMETAC brushes modified cotton.

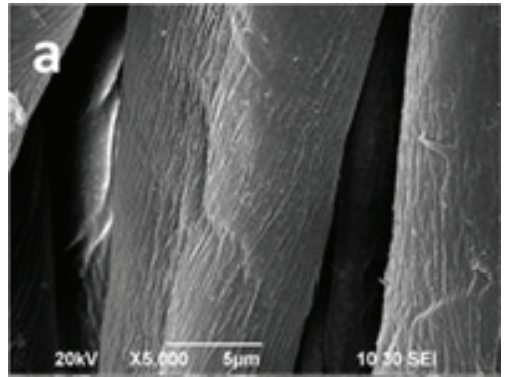

(a)

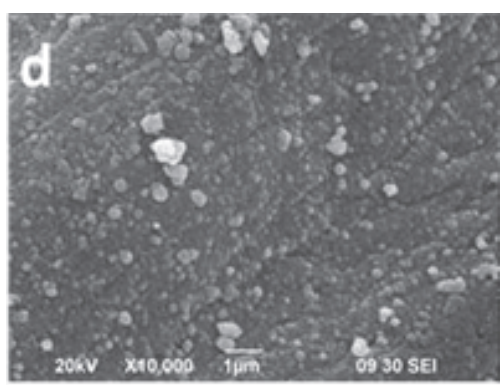

(d)

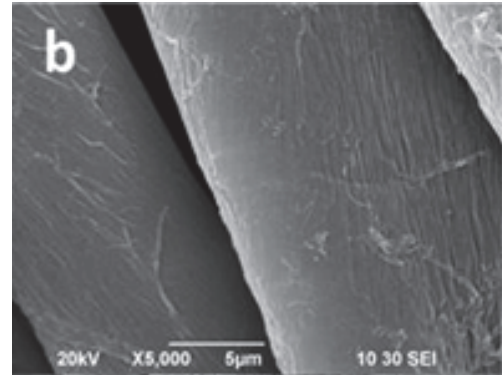

(b)

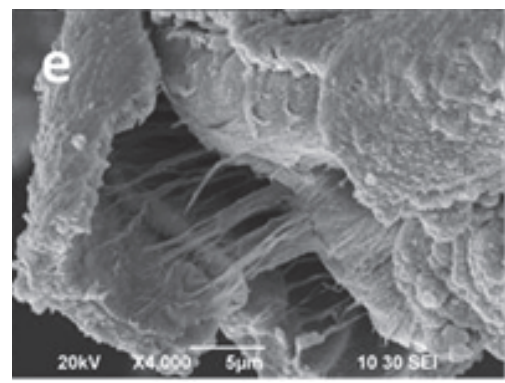

(e)

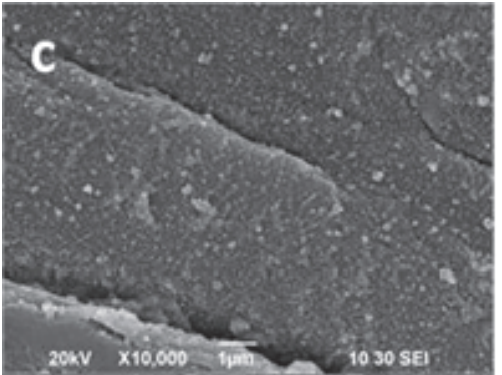

(c)

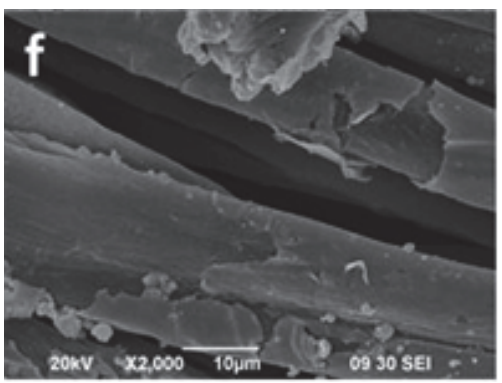

(f)

FIGURE 3: SEM images of the surface morphologies of raw cotton fibers (a), modified cotton fibers by PMETAC brushes (b), modified cotton fibers by copper nanoparticles (c), modified PET film by copper nanoparticles (d), sectional view of mechanical disruption modified cotton fibers by copper nanoparticles (e), and copper coated cotton fibers by traditional cross-linked polymer (f).

of the grafting process. The peaks of three different $\mathrm{C}-\mathrm{H}$ stretching vibrations are shown at $2853 \mathrm{~cm}^{-1}, 2919 \mathrm{~cm}^{-1}$, and $2966 \mathrm{~cm}^{-1}$.

3.2. Surface Morphologies Observation. Figure 3 shows SEM images of the surface morphologies of cotton and polymer fibers at the different treatment period. Compared with raw cotton fibers, the surface morphology of cotton fibers modified by PMETAC brushes has no obvious change (Figures 3(a) and 3(b)). It can be seen that copper particles with diameters of $130 \pm 20 \mathrm{~nm}$ were continuously and uniformly distributed on the surface of cotton fibers after the treatment process described in Experimental (Figure 3(c)). The repeatable result can be observed when the substrate is changed to polyester (Figure 3(d)). The key feature here is that those reinforced concrete-like antibacterial copper nanoparticles coatings on polymeric materials bridged by polymer brushes offer the unique stability under multiple mechanical bending, stretching, rubbing, and even washing cycles. One piece of coated fabrics was destroyed and the decoyed fiber was observed using SEM. From Figure 3(e), it can be easily discerned that the fiber surfaces are covered by the concrete-like structure composites, which contribute to the extraordinary durability and stability of antibacterial copper nanoparticles coatings 


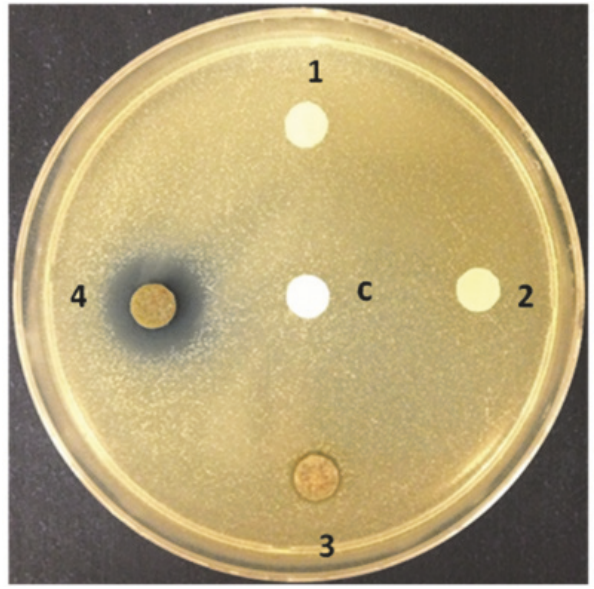

Inhibition on E. coli (ATCC 25922)

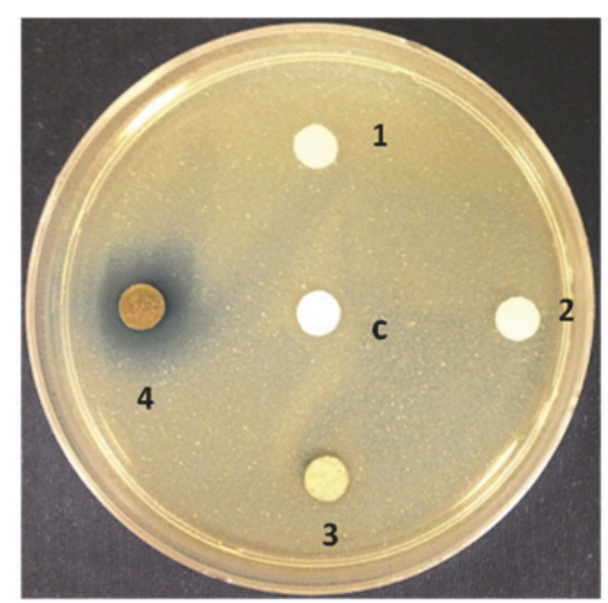

Inhibition on S. aureus (ATCC 25923)

Figure 4: Antibacterial (E. coli and S. aureus) test of control (c), modified by commercial antibacterial agents 1 (1), modified by commercial antibacterial agents 2 (2), deposited by Ag particles (3), and deposited by Cu nanoparticles (4).

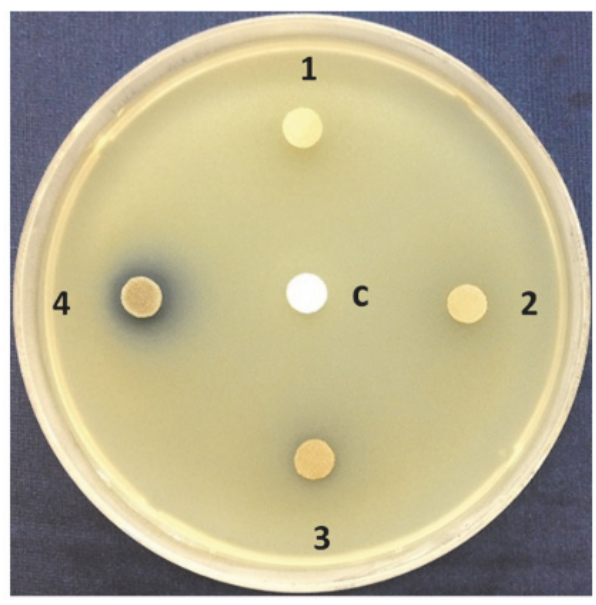

Inhibition on E. coli (ATCC 25922)

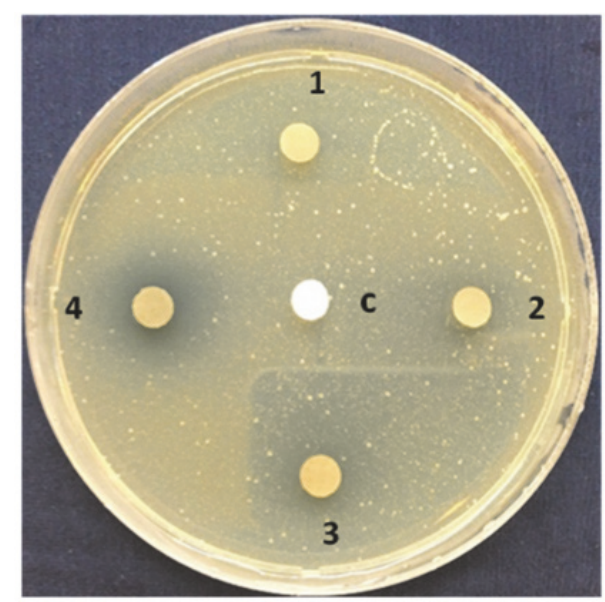

Inhibition on S. aureus (ATCC 25923)

FIgURE 5: Antibacterial (E. coli and S. aureus) test of control (c) and the samples with the different deposition time of 5 mins (1), 20 mins (2), 30 mins (3), and $1 \mathrm{~h} \mathrm{(4).}$

on polymeric materials. The copper nanoparticle layers and fibers are integrated by interfacial polymer brushes, offering the superior adhesion between $\mathrm{Cu}$ nanoparticles and substrate; therefore the polymer brushes function as "concrete iron" in the composite materials. As a comparison, commercial antibacterial cross-linked polymer and copper nanoparticles loaded on cotton fibers also were investigated under SEM and the visible disconnected coating on the fibers can be observed clearly (Figure 3(f)).

3.3. Antibacterial Activity of the Copper Nanoparticle-Modified Cotton Fabrics. In order to investigate the antibacterial activity of the copper nanoparticles-modified cotton fabrics, the control cotton fabrics, commercial antibacterial agents 1 , commercial antibacterial agents 2 , and silver particles on cotton coated by the binder were chosen as the comparative materials. Among these materials, copper nanoparticlesmodified cotton fabrics coated by ATRP and ELD show the most significant antibacterial function to $E$. coli and $S$. aureus, and the silver nanoparticles show antibacterial property, but the antibacterial ring is much smaller than that of copper (Figure 4). No evidence was observed for the two kinds of polyelectrolyte brushes modified fabrics showing antibacterial ring in this test. These results show that the deposited copper nanoparticles present the strong sterilization to the E. coli and S. aureus due to the free standing of the copper nanoparticles.

Figure 5 presents antibacterial (E. coli and S. aureus) test of control sample and the prepared coated copper samples at different deposition time. The antibacterial ring method was applied in the antibacterial test. It can be seen that raw cotton fabrics as the control sample showed the dense population of bacterial colonies, while Petri dishes, supplemented with nano-copper coating cotton fabrics at different deposition time, respectively, showed zones of inhibition around the functional specimens. The clearing zone indicates 


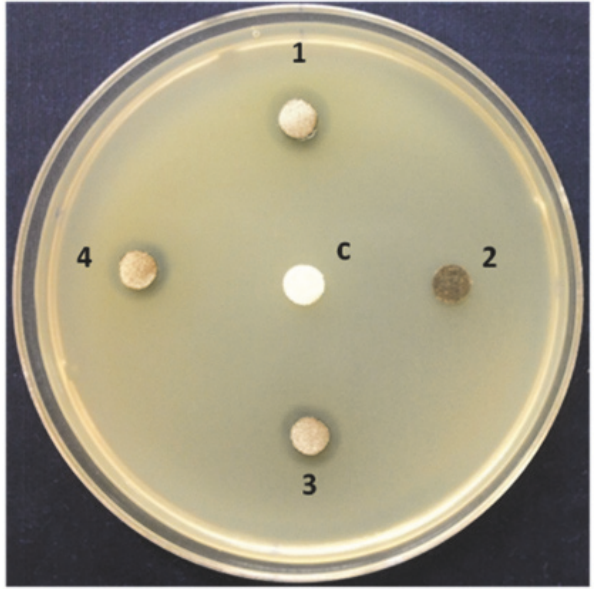

Inhibition on E. coli (ATCC 25922)

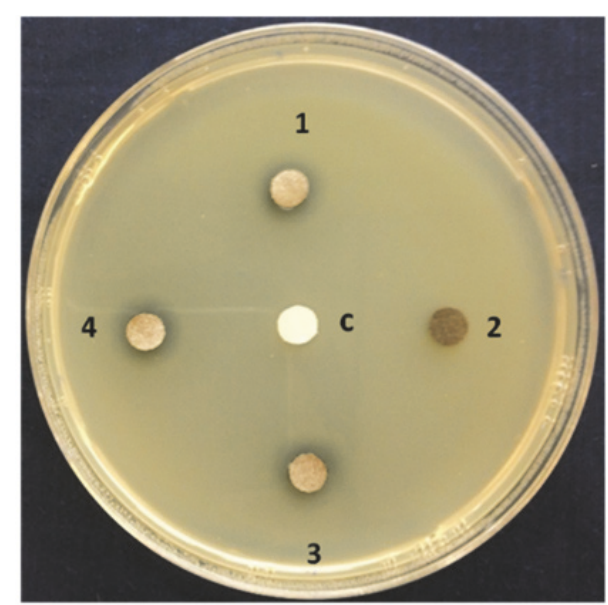

Inhibition on S. aureus (ATCC 25923)

Figure 6: Antibacterial (E. coli and S. aureus) test of control cotton fabrics without any modification (c) and the sample being unwashed (1) and the sample washed for 10 cycles (3), 30 cycles (4), and PVD-deposited copper on cotton fabrics washed for 5 cycles (2).

the susceptibility of the bacteria to the prepared sample and strong antibacterial activity of nano-copper coating cotton fabrics. What is noteworthy is that the annulus of the inhibition zone increases with the increase of the copper depositing time. In other words, the prepared fibers with more copper nanoparticles show better antibacterial properties, indicating that the copper content influences the antibacterial activity, which is similar to the previous report [21]. The remarkable antibacterial properties of copper nanoparticles-modified fibers could essentially be attributed to the biocidal action of $\mathrm{Cu}^{2+}$ ions, which are released from the functional fibers.

\subsection{Durable Testing of the Copper Nanoparticle-Modified} Cotton Fabrics. In consideration of economic costs, the durability of the antibacterial material is an important factor influencing the practical application. Apparently, the antibacterial properties of functional fabrics, fabricated by electroless deposition by polymer brushes bridged, possessed the excellent washable properties even after 30 cycles of washing, and the antibacterial ring can still be observed (Figure 6). For comparison, copper nanoparticle-coated cotton fabrics by PVD method were tested in the same disk. After even 5 cycles of washing, the PVD functional fabrics showed invisible antibacterial ring, which means that the majority of the functional copper nanoparticles were washed off because of the weak interfacial adhesion strength between copper particles and substrate. The washing testing proves that the polymer brushes-bridged-deposited copper has excellent adhesion and long durable properties, as shown in the schematic diagram of copper ions release and antibacterial performance of the functional fabrics (Figure 7).

3.5. Dynamic Release of Cu(II) from Nanocomposites. Despite the large amount of research works about the antibacterial properties of copper, the antibacterial mechanism is too complicated to reveal completely $[9,10,22]$. Undoubtedly, killing bacteria process of copper started from the $\mathrm{Cu}$ ions release. Therefore, understanding the release mechanism of
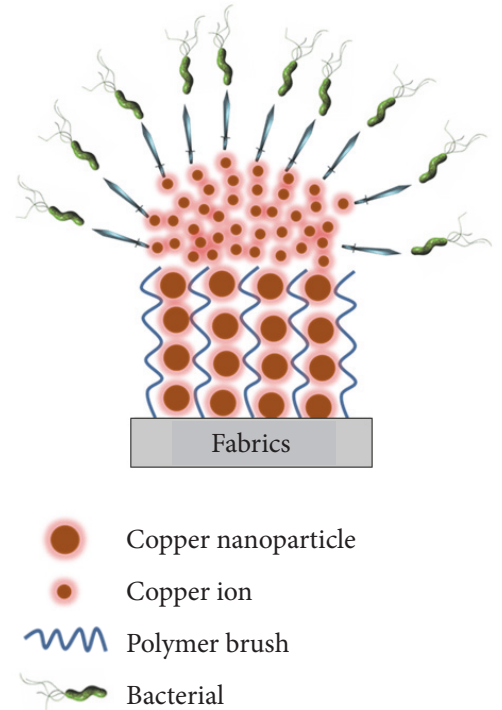

FIGURE 7: Schematic diagram of copper ions release and antibacterial performance.

copper ions is considerably important to study the novel copper-polymer antibacterial systems.

The results of $\mathrm{Cu}^{2+}$ release experiments carried out with the copper-loaded cotton fibers are shown in Figure 8. To the traditional cross-linked process, the copper nanoparticles are stuck in the cross-linked polymeric matrix, blocking the release of copper ions into the solution, and therefore copper is released in a small quantity. It is shown that the polymer brushes-bridged-deposited copper coatings more easily release the copper ion and have higher release amount. Meanwhile, as the electroless deposition time increases, the copper releases from the free standing coating also increase. This is basically due to the fact that, with the increase of the depositing time, more and more copper nanoparticles are spontaneously assembled onto surface 


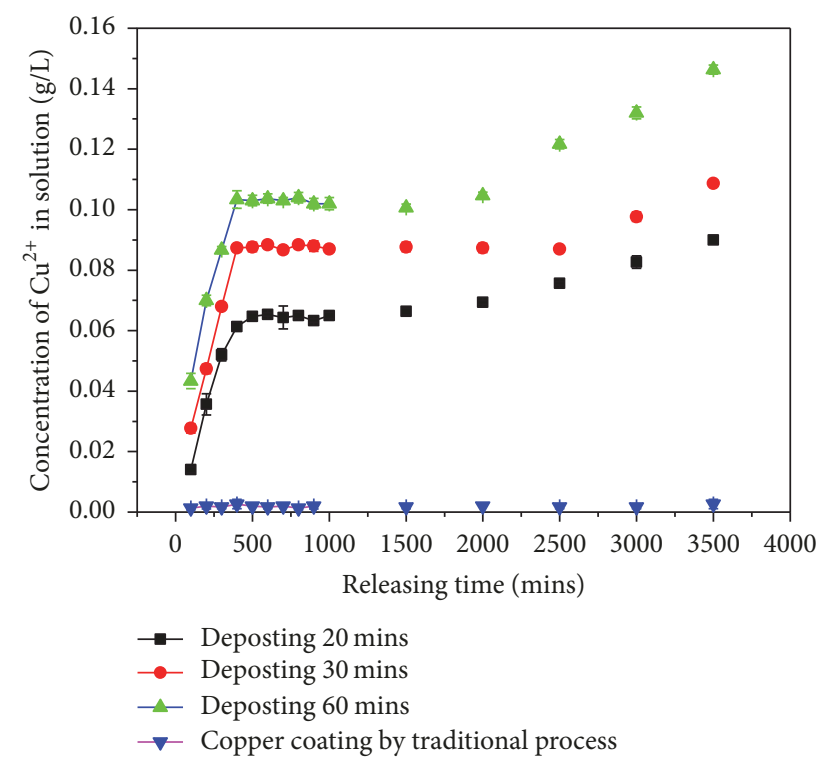

FIGURE 8: Constantly monitoring concentration of $\mathrm{Cu}^{2+}$ released from different samples against the releasing time. Reinforced concrete-like antibacterial composites with the different deposition time (20 mins, $30 \mathrm{mins}$, and $60 \mathrm{mins}$ ) and the specimen of copper coating by traditional process.

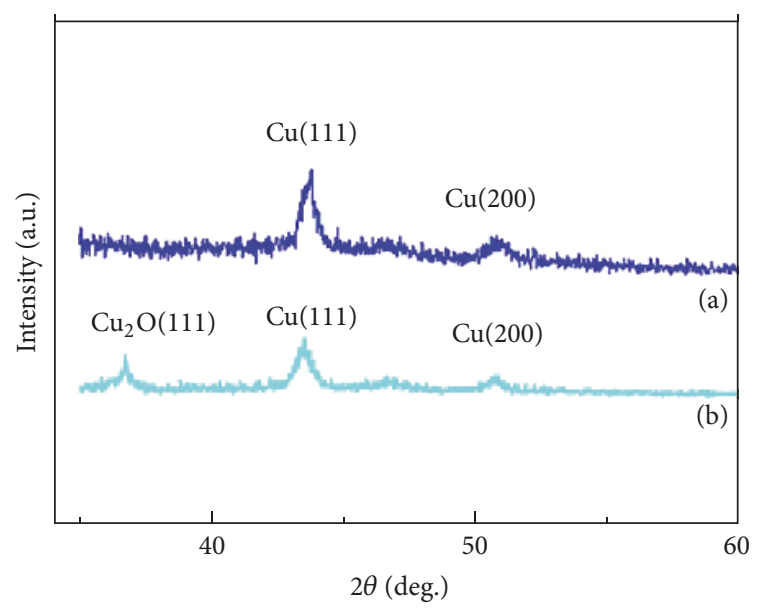

FIgURE 9: X-ray diffraction patterns of the copper coating fabrics before (a) and after (b) antibacterial test.

of polymeric materials and hence antibacterial affection improved accordingly $[8,23]$. This is consistent with the antibacterial test as a function of the deposition time in Figure 4 . Therefore, by varying the electroless deposition time, copper release and antibacterial property could be regulated.

XRD pattern analysis is utilized to examine the phase structure of $\mathrm{Cu}$. Figure 9 shows the XRD patterns of the fabrics before and after antibacterial testing for $24 \mathrm{~h}$. The functional fabrics before testing present a wide diffraction peak, indicating the formation of $\mathrm{Cu}$ nanoparticles. A new peak of $\mathrm{Cu}_{2} \mathrm{O}$ emerged on the pattern of the fabrics after the antibacterial testing. It is inferred that a small amount of $\mathrm{Cu}_{2} \mathrm{O}$ was formed in ELD process and wrapped in the $\mathrm{Cu}$ particles [24], and then antibacterial test made the copper on the outer surface release to copper ions so that the inner $\mathrm{Cu}_{2} \mathrm{O}$ was exposed and detected by XRD after test.

\section{Conclusion}

The reinforced concrete-like antibacterial copper nanoparticles coatings on cotton and polymer substrates were designed and prepared by means of ATRP and electroless deposition. $\mathrm{Cu}$ nanoparticles were continuously and uniformly distributed on the fiber surfaces of the substrate. The composite materials present excellent antibacterial ( $E$. coli and $S$. aureus) properties, even after washing by 30 cycles, and durability because of the strong interfacial force between the antibacterial copper coatings and substrates by polymer brushes. Compared with the copper coating by traditional process, the polymer brushes-bridged-deposited copper coating possessed the longer releasing effectiveness and higher releasing concentration attribute to the freestanding copper nanoparticles coatings. The controllable releases of copper ions are expected to be exploited by varying the electroless deposition time. Designed reinforced concrete-like antibacterial composite accomplishes the huge demand on modern medical and healthcare applications, which demonstrate the great potential at polymeric facilities and devices.

\section{Conflicts of Interest}

The authors declare that they have no conflicts of interest.

\section{Acknowledgments}

The authors wish to acknowledge the supporting funding from the University of Manchester (AA01906), Fundamental Research Funds for the Central Universities (31920150002), the National Natural Science Foundation of China (Grant no. 51563022), and Gansu Science and Technology Support Projects (1504GKCA093).

\section{References}

[1] D. Zhang, L. Chen, C. Zang, Y. Chen, and H. Lin, "Antibacterial cotton fabric grafted with silver nanoparticles and its excellent laundering durability," Carbohydrate Polymers, vol. 92, no. 2, pp. 2088-2094, 2013.

[2] A. M. Abdel-Mohsen, R. M. Abdel-Rahman, R. Hrdina, A. Imramovský, L. Burgert, and A. S. Aly, "Antibacterial cotton fabrics treated with core-shell nanoparticles," International Journal of Biological Macromolecules, vol. 50, no. 5, pp. 12451253, 2012.

[3] F. Zhang, X. L. Wu, Y. Y. Chen, and H. Lin, "Application of silver nanoparticles to cotton fabric as an antibacterial textile finish," Fibers and Polymers, vol. 10, no. 4, pp. 496-501, 2009.

[4] A. Jain, L. S. Duvvuri, S. Farah, N. Beyth, A. J. Domb, and W. Khan, "Antimicrobial polymers," Advanced Healthcare Materials, vol. 3, no. 12, pp. 1969-1985, 2014. 
[5] I. Perelshtein, G. Applerot, N. Perkas, G. Guibert, S. Mikhailov, and A. Gedanken, "Sonochemical coating of silver nanoparticles on textile fabrics (nylon, polyester and cotton) and their antibacterial activity," Nanotechnology, vol. 19, no. 24, Article ID 245705, 2008.

[6] S. Ravindra, Y. Murali Mohan, N. Narayana Reddy, and K. Mohana Raju, "Fabrication of antibacterial cotton fibres loaded with silver nanoparticles via 'Green Approach,' Colloids and Surfaces A: Physicochemical and Engineering Aspects, vol. 367, no. 1-3, pp. 31-40, 2010.

[7] T. Jiang, L. Liu, and J. Yao, "In situ deposition of silver nanoparticles on the cotton fabrics," Fibers and Polymers, vol. 12, no. 5, pp. 620-625, 2011.

[8] B. D. Du, D. V. Phu, L. A. Quoc, and N. Q. Hien, "Synthesis and Investigation of Antimicrobial Activity of $\mathrm{Cu}$," Journal of Nanoparticles, vol. 2017, pp. 1-6, 2017.

[9] B. Jia, Y. Mei, L. Cheng, J. Zhou, and L. Zhang, "Preparation of copper nanoparticles coated cellulose films with antibacterial properties through one-step reduction," ACS Applied Materials \& Interfaces, vol. 4, no. 6, pp. 2897-2902, 2012.

[10] R. S. Kalhapure, K. G. Akamanchi, C. Mocktar, and T. Govender, "Synthesis and antibacterial activity of silver nanoparticles capped with a carboxylic acid-terminated generation 1 oleodendrimer," Chemistry Letters, vol. 43, no. 7, pp. 1110-1112, 2014.

[11] L. Laskowski, M. Laskowska, K. Fijalkowski et al., "New Class of Antimicrobial Agents: SBA-15 Silica Containing Anchored Copper Ions," Journal of Nanomaterials, vol. 2017, Article ID 1287698, 2017.

[12] A. R. Rathmell and B. J. Wiley, "The synthesis and coating of long, thin copper nanowires to make flexible, transparent conducting films on plastic substrates," Advanced Materials, vol. 23, no. 41, pp. 4798-4803, 2011.

[13] G. Mary, S. K. Bajpai, and N. Chand, "Copper (II) ions and copper nanoparticles-loaded chemically modified cotton cellulose fibers with fair antibacterial properties," Journal of Applied Polymer Science, vol. 113, no. 2, pp. 757-766, 2009.

[14] L. A. Tamayo, P. A. Zapata, F. M. Rabagliati et al., "Antibacterial and non-cytotoxic effect of nanocomposites based in polyethylene and copper nanoparticles," Journal of Materials Science: Materials in Medicine, vol. 26, no. 3, article 129, 2015.

[15] J. Díaz-Visurraga, C. Daza, C. Pozo, A. Becerra, C. von Plessing, and A. García, "Study on antibacterial alginate-stabilized copper nanoparticles by FT-IR and 2D-IR correlation spectroscopy," International Journal of Nanomedicine, vol. 7, pp. 3597-3612, 2012.

[16] S. K. Bajpai, M. Bajpai, and L. Sharma, "Copper nanoparticles loaded alginate-impregnated cotton fabric with antibacterial properties," Journal of Applied Polymer Science, vol. 126, no. 1, pp. E318-E325, 2012.

[17] X. Liu, H. Chang, Y. Li, W. T. S. Huck, and Z. Zheng, "Polyelectrolyte-bridged metal/cotton hierarchical structures for highly durable conductive yarns," ACS Applied Materials \& Interfaces, vol. 2, no. 2, pp. 529-535, 2010.

[18] X. Liu, Y. Li, Z. Zheng, and F. Zhou, "Ionic liquids as twodimensional templates for the spontaneous assembly of copper nanoparticles into nanobelts and observation of an intermediate state," RSC Advances, vol. 3, no. 2, pp. 341-344, 2013.

[19] X. Liu, Y. Li, J. Hu, J. Jiao, and J. Li, “Smart moisture management and thermoregulation properties of stimuli-responsive cotton modified with polymer brushes," RSC Advances, vol. 4, no. 109, pp. 63691-63695, 2014.
[20] D. A. Steinberg and R. I. Lehrer, Antibacterial Peptide Protocols, W. M. Shafer, Ed., vol. 78 of Methods In Molecular Biology, Humana Press, Totowa, NJ, USA, 1997, 169-186.

[21] J. Liu, F. Li, C. Liu et al., "Effect of Cu content on the antibacterial activity of titanium-copper sintered alloys," Materials Science and Engineering C: Materials for Biological Applications, vol. 35, no. 1, pp. 392-400, 2014.

[22] A. K. Chatterjee, R. Chakraborty, and T. Basu, "Mechanism of antibacterial activity of copper nanoparticles," Nanotechnology, vol. 25, no. 13, Article ID 135101, 12 pages, 2014.

[23] M. Raffi, S. Mehrwan, T. M. Bhatti et al., "Investigations into the antibacterial behavior of copper nanoparticles against Escherichia coli," Annals of Microbiology, vol. 60, no. 1, pp. 75-80, 2010.

[24] J. J. Kim, S. H. Cha, and Y.-S. Lee, "Seedless fill-up of the damascence structure only by copper electroless plating," Japanese Journal of Applied Physics, vol. 42, no. 8 A, pp. L953-L955, 2003. 


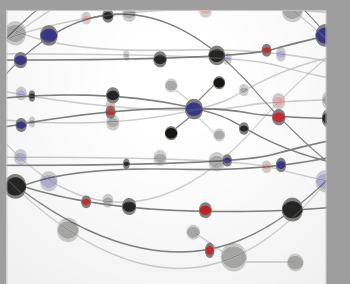

The Scientific World Journal
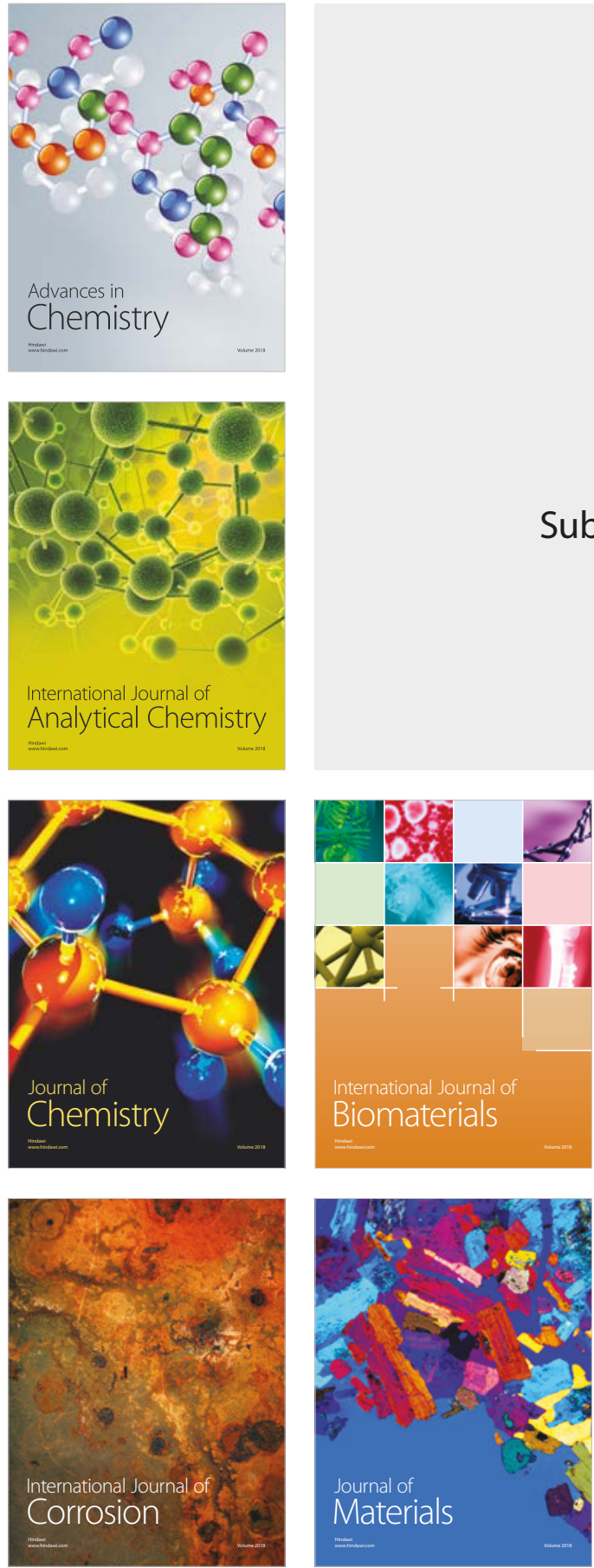

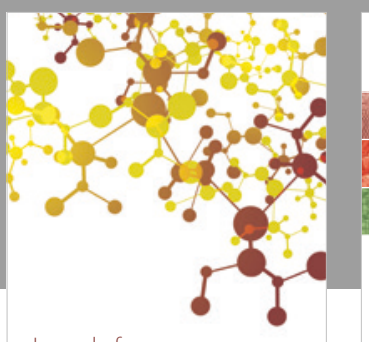

Journal of

Applied Chemistry
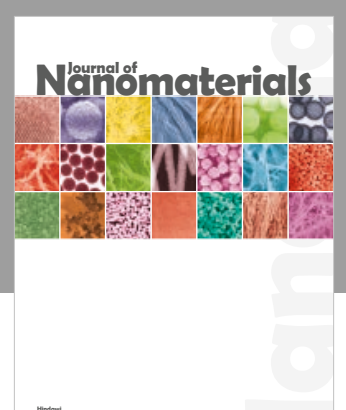

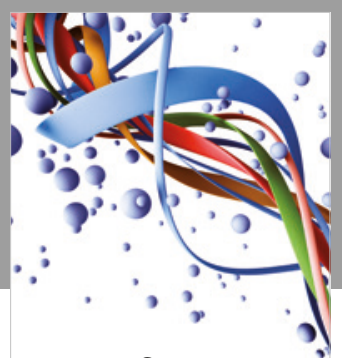

Scientifica

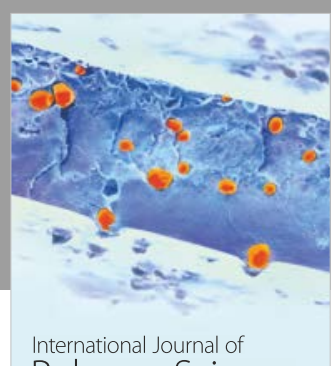

Polymer Science

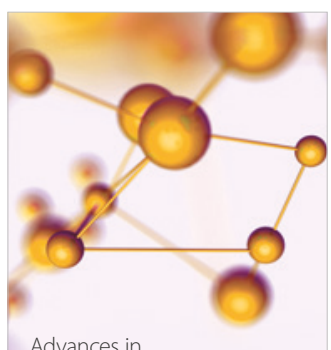

Physical Chemistry
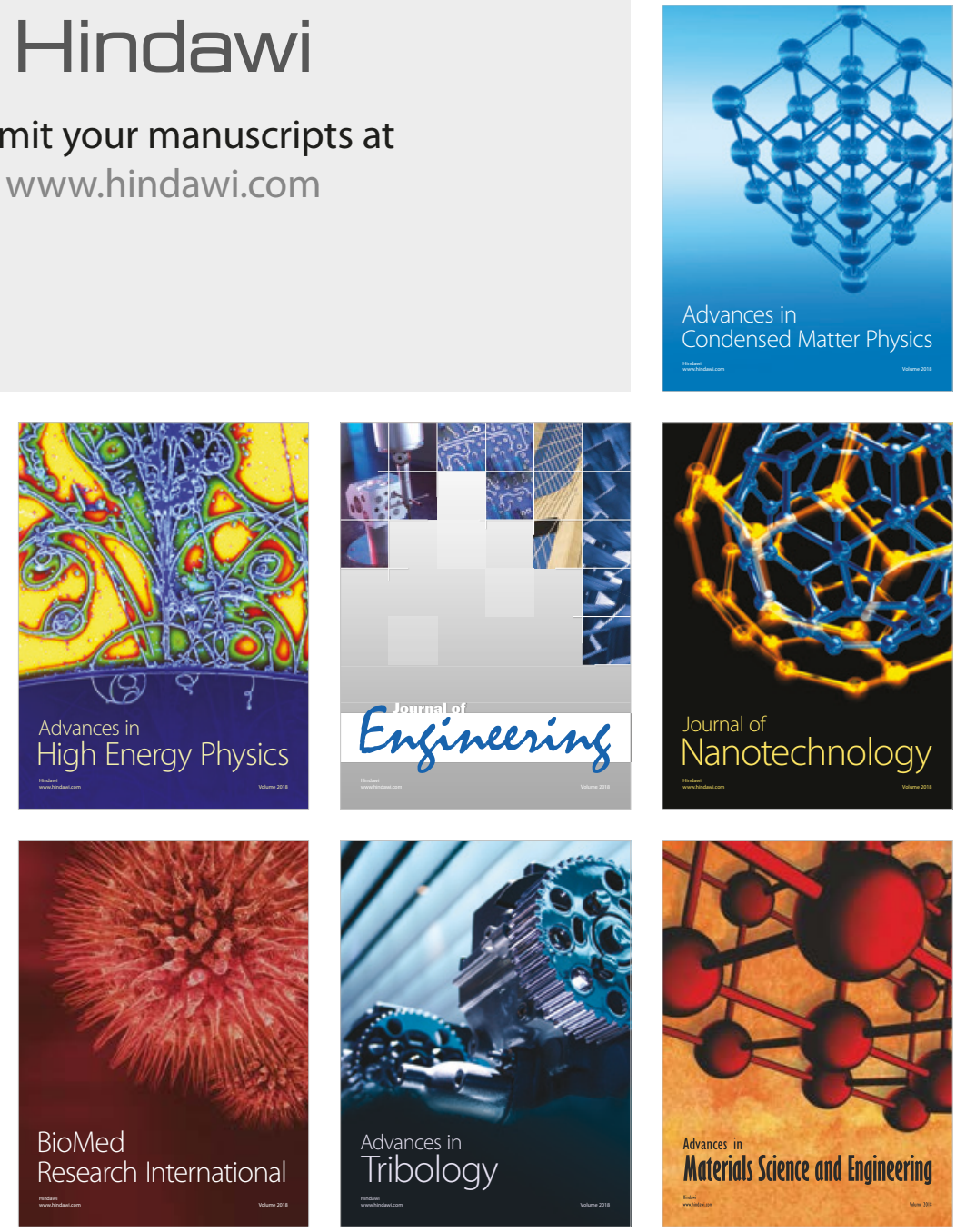